\title{
Characterization of Gas Permeable Template Material for Nanoimprinting
}

\author{
Naoto Sugino ${ }^{1,2^{*}}$, Satoshi Takei ${ }^{1}$, Shinya Nakajima ${ }^{1}$, Makoto Hanabata ${ }^{1}$, \\ Takao Kameda ${ }^{2}$, and Atsushi Sekiguchi ${ }^{3}$ \\ ${ }^{1}$ Department of Mechanical Systems Engineering, Toyama Prefectural University, \\ Imizu, Toyama 939-0398 Japan \\ ${ }^{2}$ Technology Development Division, SANKO GOSEI Ltd. 4185 Koreyasu Nanto \\ Toyama 939-1852 Japan \\ ${ }^{3}$ Litho Tech Japan Corporation, Saitama 332-0034, Japan \\ *n.sugino@sankogosei.co.jp
}

\begin{abstract}
In recent years, optical lithography technology used for micro fabrication of electronic devices, semiconductors, etc. has continues to advance for the purpose of cost reduction and improvement. However, plenty of transfer failure caused by air entrained when pressing the mold against the material to be transferred or gas generated from the resist material were reported in the nanoimprint process. Therefore, in order to develop a novel template material with gas permeability, chemical modification of crystalline cellulose with different viscosity and molecular weight was evaluated the physical properties in this study. By using this gas permeable template material as a mold, a transfer material containing acetone as a volatile solvent was molded. As a result, a shape of $5 \mu \mathrm{m}$ was transferred to be the material containing a volatile solvent, and no defect due to a gas generated from a volatile solvent was confirmed. This gas permeable template material can be expected to reduce transfer defects in imprints and can be said to have a possibility to broaden the selectivity of resist materials.
\end{abstract}

Keywords: Nanoimprint lithography, Hydroxypropyl cellulose, Indentation modulus, Light transmittance, Gas permeability

\section{Introduction}

In recent years, nanoimprint lithography using nanotechnology has been used in the fabrication of electronic parts such as LSI, memory, and hard disk $[1,2]$. The nanoimprint lithography is a processing method proposed by Chou et al. of Princeton University, and it was reported that it was succeeded in transcription of $10 \mathrm{~nm}$ [3-5]. The method proposed by Chou et al. was called thermal nanoimprint lithography because it is used in a process of heating and softening a resist material, cooling it after shaping, and then cooling. Furthermore, a method called photo nanoimprint lithography in which a photo curable resin is used as a resist material and is cured by ultraviolet rays for shaping is proposed by Willson of the University of Texas [6]. This method has reported that the transfer of 2 to $3 \mathrm{~nm}$ [7] and the difference in line edge roughness was $0.1 \mathrm{~nm}$ [8]. The nanoimprint lithography is extremely useful because it has a feature that it can be processed cost-effectively and efficiently than conventional exposure apparatuses. However, Figure 1 a) shows that the air or gas entrapped between the template and the material to be transferred when pressing the template against the material to be transferred, b) volatile gases generated from additives of the resist material, or reaction gas generated by heat or light reaction, an important problem on the template is that the shape of the transfer material is not transferred to the transfer material has been reported [9-11]. Gas and solvent permeability templates have also been reported to solve transfer defects and template damage caused by gases such as nitrogen and oxygen generated from materials [12-14]. Therefore, in order to solve the transfer defect due to air and gas entrapped between the template and the transferred material, we reported the development of a template material that permeates the gas using the plant-derived material in the previous research 
[15-19]. The evaluation of the mechanical strength in the gas permeable template material was required for high volume manufacturing. Therefore, the purpose of this research is to investigate the dependence of weight average molecular weight on the properties such as mechanical strength and gas permeability of gas permeable template materials. Cellulose derivatives were prepared by changing the viscosity of hydroxypropyl cellulose as the main component of the gas permeable template material, and their mechanical strength, gas permeability and light transmittance were evaluated. A volatile solvent was included in the material to be transferred, and the transferability and gas permeability of the template material were confirmed.

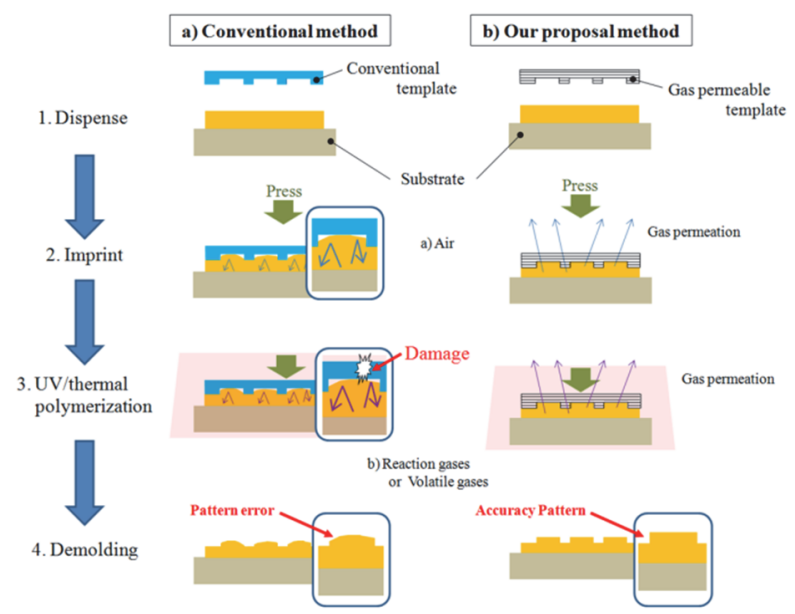

Fig.1. Comparison of a) conventional method and b) our proposal method.

\section{Experimental}

2.1 Raw material for gas permeable template material

Figure 2 shows the chemical structural formula of hydroxypropyl cellulose (HPC) which is the main component of the cellulose derivative. Three HPC with different weight average molecular weights $(M \mathrm{w})$ (viscosities) were prepared in the experiment. Three different viscosities of HPC were 2.0-2.9, 3.0-5.9, and $6.0-10 \mathrm{mPa} \cdot \mathrm{s}$, respectively.

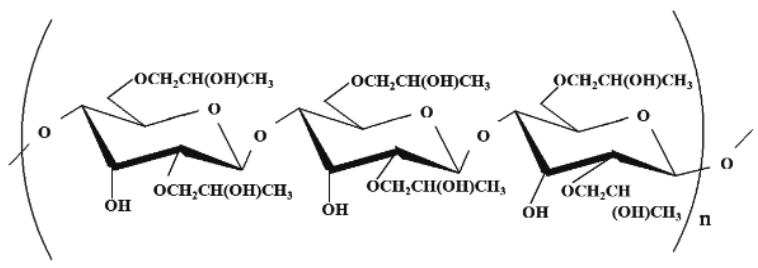

Fig. 2. Chemical structural formula of hydroxypropyl cellulose (HPC).
2-Methacryloyloxyethyl isocyanate (MOI) having an isocyanate group and double bond was used as a crosslinking agent, 2-butanone was used as a solvent, and triethylamine was used as a synthetic reaction initiator.

2.2. Measurement of molecular weight distribution of HPC

Molecular weights of HPC with different viscosities were measured by using the GPC (HLC8320 GPCEcoSEC, Tosoh). The conditions of the GPC uses one separation column of SuperAW3000 and two separation columns of SuperAW-H (TSKGEL, Tosoh) and $N, N$-dimethylformamide. A column temperature was maintained at $40{ }^{\circ} \mathrm{C}$ and the solvent flow rate was $0.6 \mathrm{~mL} / \mathrm{min}$. In addition, polystyrene (PS) (PStQuick Tosoh) was used as a standard sample for preparing a calibration curve. This standard sample consists of 210000, 397000, 37200, 6200, and 589 number-average molecular weight $(M \mathrm{w})$.

The measured results were shown in Table 1. It was confirmed that the number-average molecular weight $(M \mathrm{w})$ increased as the viscosity increased.

Table 1. Result of molecular weight distribution of HPC

\begin{tabular}{|l|l|l|l|}
\hline Viscosity & $\begin{array}{l}2.0-2.9 \\
\mathrm{mPa} \cdot \mathrm{s}\end{array}$ & $\begin{array}{l}3.0-5.9 \\
\mathrm{mPa} \cdot \mathrm{s}\end{array}$ & $\begin{array}{l}6.0-10 \\
\mathrm{mPa} \cdot \mathrm{s}\end{array}$ \\
\hline$M \mathrm{n}$ & 18,800 & 36,700 & 47,800 \\
\hline$M \mathrm{w}$ & 33,350 & 68,900 & 88,700 \\
\hline
\end{tabular}

2.3. Synthesis of cellulose derivatives in gas permeable template materials

Figure 3 shows a method for synthesizing a cellulose derivative in the gas permeable template material. The HPC was added 2-butanone and mixed at $70{ }^{\circ} \mathrm{C}$ for 60 minutes. And, MOI was added to it and mixed at $60 \pm 5^{\circ} \mathrm{C}$ for 20 minutes. The solid content concentration of HPC in the synthesized cellulose derivative was $20 \mathrm{wt} \%$. It was confirmed by FT-IR analysis that HPC and MOI were chemically synthesized by this synthesis method. Then, triethylamine was added to the cellulose derivative at a concentration of $8.9 \mathrm{wt} \%$ and stirred in a 1,1,1,2-tetrafluoroethane atmosphere at $60{ }^{\circ} \mathrm{C}$ for 80 minutes.

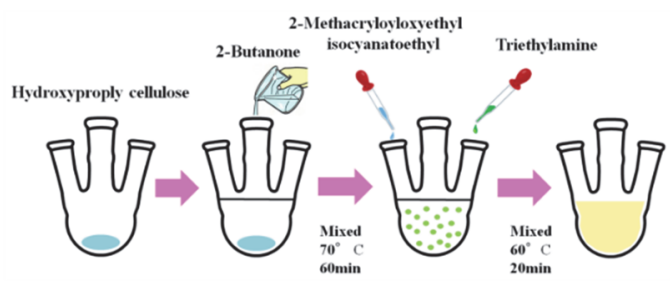

Fig. 3. Method for synthesizing cellulose derivative. 
2.4. Preparation of gas permeable template material with cellulose derivatives

The gas permeable template material was prepared by adding AIBN which is a thermal radical initiator to the cellulose derivative and mixing for 15 minutes. The concentrations of the cellulose derivative and AIBN were 97 and $3 \mathrm{wt} \%$, respectively. The mixed sample was coated on a glass plate and subjected to a deaeration treatment at room temperature for 1 hour under a reduced pressure of $0.1 \mathrm{MPa}$, further depressurized to 0.04 $\mathrm{MPa}$, and dried at $110^{\circ} \mathrm{C}$ for 2 hours.

2.5. Mechanical properties of gas permeable template material

The indentation elastic modulus was measured by a nanoindentation test. As a measuring machine, a dynamic ultra-micro hardness tester DUH-211 (Shimadzu Corporation) was used, and a Berkovich indenter was used as an indenter. A load-unloading test with a test force of $30 \mathrm{mN}$ was performed, five positions were measured by changing the position of one sample, and the indentation elastic modulus was calculated from the average value.

\subsection{Light transmittance of gas permeable template material}

Light transmittance of the gas permeable template materials were measured using a UV-visible light spectrophotometer V-650 (JASCO). The gas permeable template material was applied to a flat glass plate and dried under reduced pressure at $110^{\circ} \mathrm{C}$ for 2 hours. Then, in the wavelength range of 300 to $900 \mathrm{~nm}$, the data reading interval was set to $1.0 \mathrm{~nm}$ at a scanning speed of $400 \mathrm{~nm} / \mathrm{min}$, and the optical transparency of the film was measured. The measured spectrum was calculated by subtracting the spectral difference of only the glass plate.

\subsection{Gas permeability of gas permeable template} material

Gas permeability of that gas permeable template materials were measured by GTR-10 ATU (GTR Tec). A gas chromatograph (G 2700 T Yanaco Anal.) was used as a detector for the transmitted gas. The thickness of the film was about $250 \mu \mathrm{m}$, oxygen gas was used as the test gas, and the measurement temperature was $30^{\circ} \mathrm{C}$.

2.8. Preparation of template using gas permeable template material

Since the solid content concentration of HPC of the cellulose derivative was $20 \mathrm{wt} \%$, the solid content concentration was adjusted to $54 \mathrm{wt} \%$ or more by vacuum drying at room temperature. Thereafter, AIBN as a heat hardening agent was mixed at a concentration of $3 \mathrm{wt} \%$ to prepare a template material.

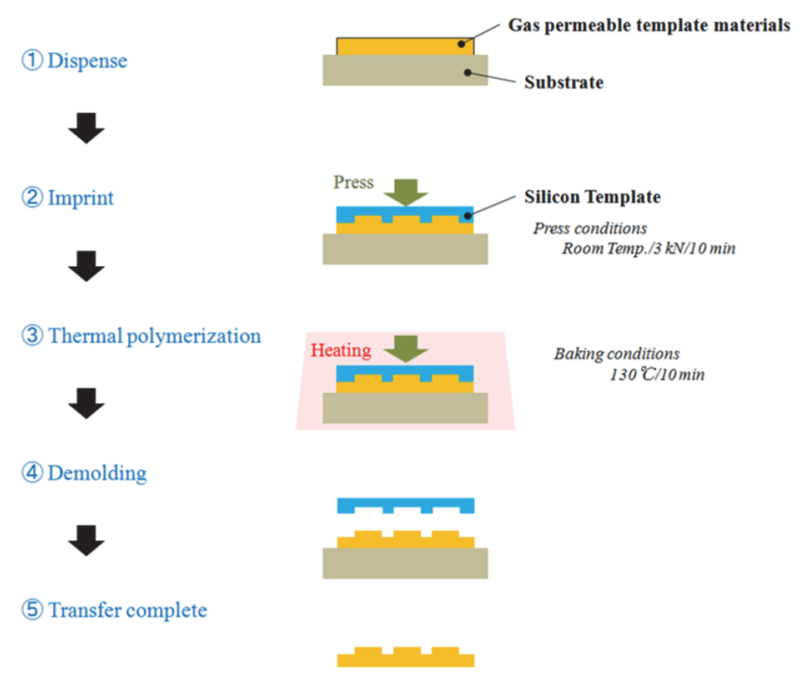

Fig. 4. Procedure for preparing gas permeable template.

Figure 4 shows a procedure for preparing a template using gas permeable template material. The prepared template material was applied on a substrate and a silicon template $(20 \mathrm{~mm} \times 20 \mathrm{~mm})$ having a $5 \mu \mathrm{m}$ line and space shape was pressed at room temperature under a pressure of $3 \mathrm{kN}$ for 10 minutes. The template were dried in a dryer at $130{ }^{\circ} \mathrm{C}$ for 10 minutes. The surface of the template was observed with a confocal microscope (OPTELICS H1200, Lasertec). And the imprint tester (LTNIP-5000, Litho Tech Japan) was used for preparing a template using gas permeable template material.

\section{Results and discussion}

3.1. Mechanical properties of gas permeable template material

Figure 5 shows the dependence of indentation modulus on HPC molecular weight. As the molecular weight of HPC increased, indentation elastic modulus decreased. HPC with short main chain seems to be likely to aggregate and formed a high density network by crosslinking reaction.

3.2. Light transmittance of gas permeable template material

Figure 6 shows the dependence of light transmittance on the average molecular weight of 
HPC. The wavelength used for UV nanoimprint lithography was $365 \mathrm{~nm}$. Even if the average molecular weight of HPC increased, the light transmission hardly changed. Regardless of the average molecular weight of the HPC, the crosslinked molecules seem to have undergone a crosslinking reaction with the HPC while maintaining a certain interval. Since the HPC itself has light transparency, it is inferred that the optical transparency of the HPC film forming a high density network also increased.

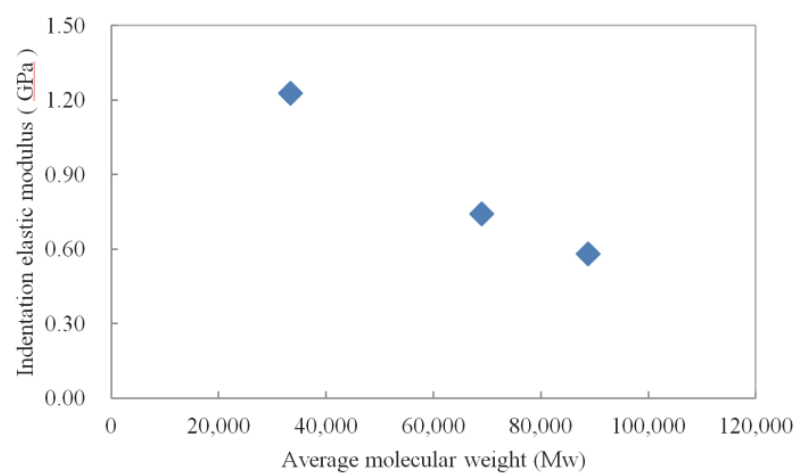

Fig. 5. Average molecular weight of HPC dependence of indentation elastic modulus of permeable template material.

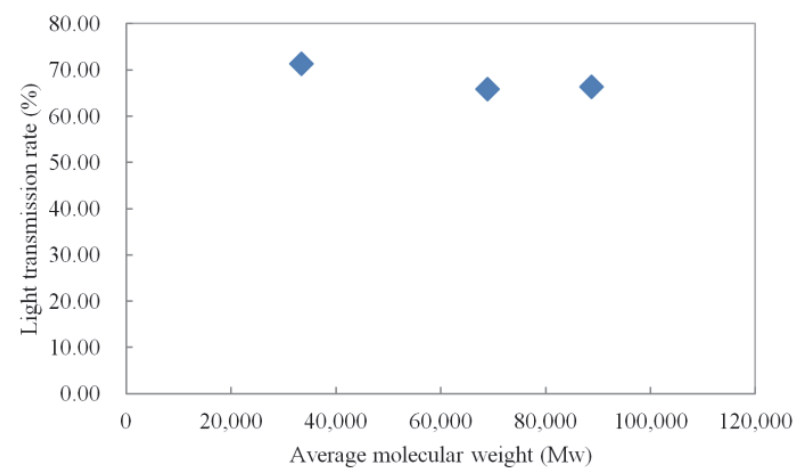

Fig. 6. Average molecular weight of HPC dependence of the light transmission rate of permeable template material.

\subsection{Gas permeability of gas permeable template} material

Figure 7 shows the dependence of gas permeability on the average molecular weight of HPC. As the average molecular weight of HPC increased, gas permeability also increased. HPC with short main chain are denser and there seems to be no gap through which gas passes. In order to confirm these, it is necessary to measure the size of the gap through which the gas can penetrate due to the difference in molecular weight. In other words, based on the results in Figure 5, it can be said that the elastic modulus of the permeable template material and the gas permeability are in a trade-off relationship.

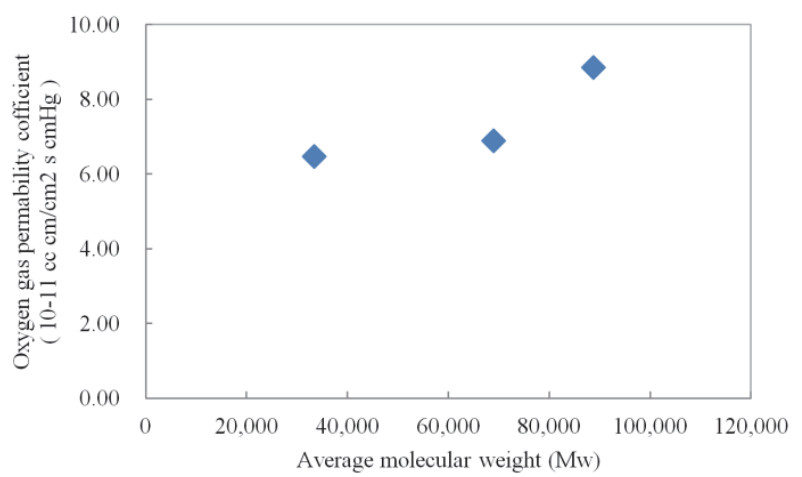

Fig. 7. Average molecular weight of HPC dependence of the oxygen gas permeability coefficient of permeable template material.

3.4. Transcription of gas permeable template material

Figure 8 shows a confocal micrograph of the gas permeable template surface at each molecular weight. This shows that structures of the master template were exactly transferred to gas permeable template. It can also be said that there is no change in transferability depending on the molecular weight. If the indentation modulus of the template is $0.58 \mathrm{GPa}$ or more, it can be said that a shape of 5 $\mu \mathrm{m}$ can be transferred to the template.
(A) $M \mathrm{w}=33,350$
(B) $M \mathrm{w}=68,900$
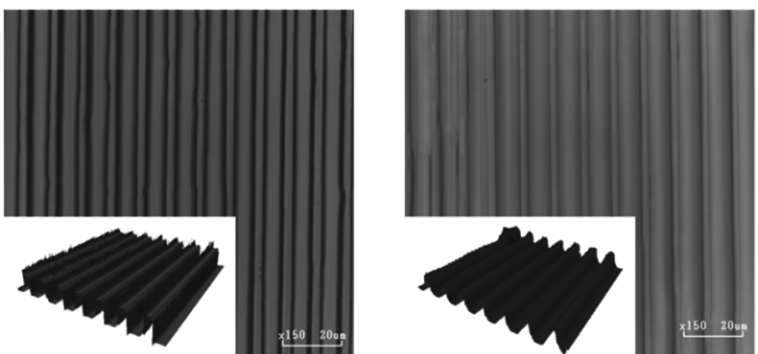

(C) $M \mathrm{w}=88,700$

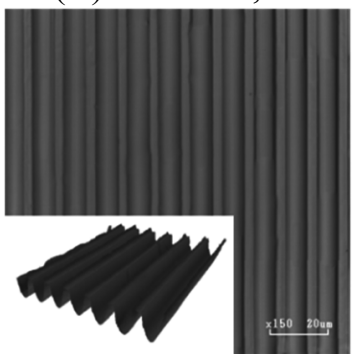

Fig. 8. Micrographs of patterned of $5 \mu \mathrm{m}$ line regularlynanostructures in gas permeable template.

For the purpose of evaluating gas permeability of gas permeable template in imprint lithography 
process, a gas permeable template was made with HPC with the lowest gas permeability. The prepared gas permeable template with patterned of $5 \mu \mathrm{m}$ line and spaces regularly-nanostructures was imprinted to a UV cross-linked liquid material containing a volatile solvent. The UV cross linked liquid material contains acetone as a volatile solvent at $5 \mathrm{wt} \%$ concentration. Figure 9 shows that the process of imprint lithography.

Since the solid content concentration of the gas permeable template material to be used is $20 \%$, the solid content concentration of the gas permeable template material was concentrated to $60 \%$ under reduced pressure drying at $30^{\circ} \mathrm{C}$.

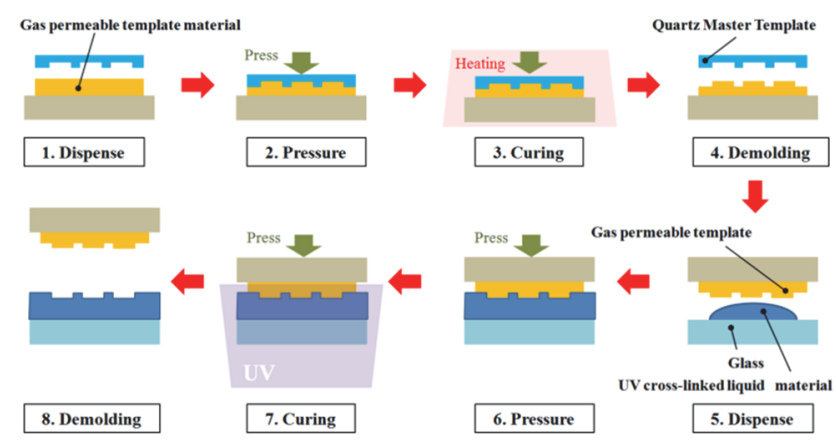

Fig. 9. Process of imprint lithography.

(1) Quartz master template

(2) Gas permeable template
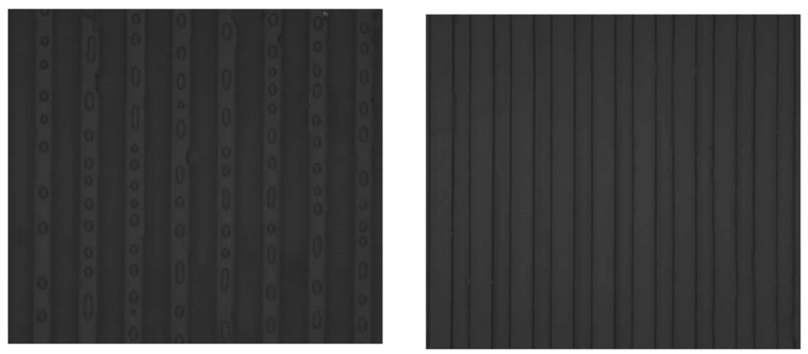

Fig. 10. Confocal microscopic image of UV-crosslinked liquid material imprinted by using (1) quartz master template and (2) gas permeable template.

Figure 10 shows a confocal microscopic image of UV-crosslinked liquid material containing acetone imprinted using (1) quartz master template and (2) gas permeable template. Figure 10-(1), there are air bubbles in the UV-crosslinked liquid material because of the gas volatilized from acetone, Figure 10-(2) there is no bubble in the UV-crosslinked liquid material. In other words, transfer to the UVcrosslinked liquid material in the gas permeable template was successful. In the case of a material containing $5 \mathrm{wt} \%$ of acetone, it means that it can transfer if it has a gas permeability of $6.48 \times 10^{-11}$ $\mathrm{cc} \cdot \mathrm{cm} / \mathrm{cm}^{2} \cdot \mathrm{s} \cdot \mathrm{cmHg}$. In future research, it's also necessary to investigate volatile solvents other than acetone.

\section{Conclusions}

The indentation modulus of the gas permeable template decreases with increasing molecular weight. On the other hand, the gas permeability of the gas permeable template increases with increasing molecular weight. It is in a trade-off relationship.

The gas permeable template transmits gas generated from the solvent contained in the transfer material. It is expected that the gas permeable template can contribute to reduction of transfer failure in UV or thermal imprint lithography.

\section{Acknowledgements}

This work was supported by JSPS Grant-in-Aid for scientific Research No. 16K04920, JSPS Bilateral Joint Research Projects, and Regional Innovation Strategy Support Program, Toyama Nanotech Cluster 2014 of Ministry of Education, Culture, Sports, Science and Technology, Japan. This project was also partially supported by The Canon Foundation, The Die and Mould Technology Promotion Foundation, The Amada Foundation, The Naito Taisyun Science and Technology Foundation, and The Takeuchi MFG Foundation.

\section{References}

1. S. Y. Chou, P. R. Krauss, and P. J. Renstrom, Appl. Phys. Lett., 76 (1995) 3114.

2. Y. Ishii and J. Taniguchi, Microelectron. Eng., 84 (2007) 912.

3. S. Y. Chou, P. R. Krauss, and P. J. Renstrom, Appl. Phys. Lett., 67 (1995) 3114.

4. S. Y. Chou, P. R. Krauss, and P. J. Renstrom, Science, 85 (1996) 272.

5. S. Y. Chou, P. R. Krauss, and P. J. Renstrom, J. Vac. Sci. Technol. B, 14 (1996) 4129.

6. T. Bailey, B. J. Choi, M. Colburn, M. Meissl, S. Shaya, J. G. Ekerdt, S. V. Sreenivasan, and C. G. Willson, J. Vac. Sci. Technol. B, 18 (2000) 3572.

7. F. Hua, Y. Sun, A. Gaur, M. A. Meitl, L. Bilhaut, L. Rotkina, J. Wang, P. Geil, M. Shim, and J. A. Rogers, Nano Lett., 4 (2004) 2467.

8. Y. Kurashima, H. Hiroshima, M. Komuro, N. Yamazaki, J. Taniguchi, and I. Miyamoto, Jpn. J. Appl. Phys., 42 (2003) 3871.

9. S. H. Ahn and J. S. Guo, ACS Nano, 3 (2009) 
2304.

10. S. Y. Chou, P. R. Krauss, and P. J. Renstrom, $J$. Vac. Sci. Technol. B, 14, (1996) 4159.

11. J. G. Kim, Y. Sim, Y. Cho, J. W. Seo, S. Kwon, J. W. Park, H. G. Choi, H. Kim, and S. Lee, Microelectron. Eng., 86 (2009) 2427.

12. B. Yu, H. Cong, Z. Li, H. Yuan, Q. Peng, M. Chi, S. Yang, R. Yang, R. Wickramasinghe, and J. Tang, J. Polym. Sci. Part B: Polym. Phys., 53 (2015) 552.

13. V.-T. Bui, H.-S. Lee, J.-H. Choi, and H.-S. Choi, Data Brief, 5 (2015) 990.

14. V. -T. Bui, H. -S. Lee, J. -H. Choi, and H. -S. Choi, J. Colloid Interface Sci., 331 (2009) 446.

15. S. Takei, S. Nakajima, K. Sugahara, M.
Hanabata, Y. Matsumoto, and A. Sekiguchi, Macromol. Mater. Eng., 301 (2016) 902.

16. S. Nakajima, S. Takei, Z. Zhou, H. Maki, K. Sugahara, M. Hanabata, Y. Matsumoto, and A. Sekiguchi, J. Photopolym. Sci. Technol., 29 (2016) 189.

17. S. Takei and A. Sekiguchi, Appl. Sci., 2 (2012) 24.

18. N. Sugino, S. Nakajima, T. Kameda, S. Takei, and M. Hanabata, Proc. SPIE, 9985 (2016) 99851I.

19. S. Takei, N. Sugino, T. Kameda, S. Nakajima, and M. Hanabata, Proc. SPIE, 9985 (2016) 99852C. 